\title{
Problem solving in the rat: Stay vs. shift solutions on the three-table task
}

\author{
THOM HERRMANN, ELEANOR BAHR, and BECKY BREMNER \\ University of Guelph, Guelph, Ontario NIG 2Wl, Canada \\ and \\ PAUL ELLEN \\ Georgia State University, Atlanta, Georgia 30303
}

\begin{abstract}
Two experiments were conducted to determine the conditions under which a shift problem vs. a stay problem would be easier for animals tested on Maier's three-table task. When animals were given a prior exploratory experience and a partial feeding during the feeding experience, the stay problem was easier. With a prior exploratory experience and a complete feeding experience, the shift solution was easier. In the absence of prior exploration, rats cannot solve either the stay or shift problem, regardless of the amount of feeding during the feeding experience.
\end{abstract}

Prominent among the tasks that have been highlighted as being particularly revealing in the analysis of spatial behavior are the Olton eight-arm radial maze (Olton, 1979; Olton \& Samuelson, 1976) and the Maier three-table task (Maier, 1932a). On the eight-arm maze, eight runways lead away from a center platform like spokes on a wheel. Food is placed at the end of each arm, and food-deprived rats are placed on the center platform. Their task is to choose among the eight arms until all of the food is obtained. The optimum strategy for the animals is to choose each arm once and not to repeat a choice to any arm. This is a relatively simple task inasmuch as by the completion of 40 tests the animals are choosing, on the average, 7.9 arms in the first eight choices that they make. According to Olton (1978), the response requirement in this task can be characterized as a win-shift strategy. Having found food in one location or arm, the animal should choose another arm since food will not be replaced in the original arm until the test has been completed.

In contrast with the eight-arm maze, the threetable task involves a different type of response requirement. In this task, the animals are first allowed to freely explore three unbaited tables and their interconnecting runways. They are then fed on one of the tables. After the feeding, the animals are placed on one of the two remaining tables (start table) and their task is to return to the table on which they have been fed. In other words, ideal performance on this task would favor a stay type of strategy. Each day the

Research supported in part by a grant from the National Science and Engineering Research Council of Canada. Send requests for reprints to Paul Ellen, Department of Psychology, Georgia State University, University Plaza, Atlanta, Georgia 30303. animals are given the preliminary exploratory experience, and the baited and start tables are varied so that it is not possible for the animals to learn that a given table or turning response is consistently associated with the food. Thus, the three-table task can be considered to differ from the eight-arm task in two important procedural respects. First, the optimal strategy is different in each task and, second, the three-table task involves a preliminary exploratory period each day before the feeding, whereas in the eight-arm maze this daily exploratory period is not used (Olton \& Samuelson, 1976).

Although there are procedural differences between the two tasks in terms of the response requirement, there is a common element in that both tasks involve some form of spatial memory. In the eight-arm maze, all eight alternatives are present on every trial and the animal must remember all of its previous choices in order not to repeat a choice. In the three-table task, the animal must remember on which table it has just been fed when it is placed on the starting table for its daily test trial. The question then arises as to whether, despite the procedural differences, the two tasks are functionally equivalent in terms of their underlying psychological mechanisms.

In this regard, there are relatively few data available. Winocur (1980) has shown that neocortical damage is relatively innocuous in its effects on eightarm-maze performance, and Maier (1932a) has shown that as little as $11 \%$ of such damage can severely impair three-table task performance. This difference in the effects of brain damage would suggest that, despite the fact that both tasks may involve a spatial memory factor, there is still a major distinction between them in terms of underlying psychological processes. Before this can be established, it remains 
to be determined whether other manipulations that affect eight-arm-maze performance have a comparable effect on three-table performance. To the extent that common manipulations have different outcomes, we would have further evidence for a major distinction between the psychological processes reflected by performance on these two spatial tasks. The present study is concerned with exploring this issue.

\section{EXPERIMENT 1}

Correct performance on the eight-arm maze requires that animals shift their choices after entering a baited alley and retrieving the food there. Olton (Olton, Handelmann, \& Walker, 1980; Olton \& Schlosberg, 1978) has suggested that rats have a predisposition to follow a shift strategy and not return to a place where they have just found food. Thus, the eight-arm maze is readily learned since the animal's predisposition is to shift after having just found food, and this predisposition is congruent with the optimal strategy on the task.

To what extent does this predisposition to shift following the finding of food in a given location influence performance on the three-table task? In the typical configuration of the three-table task, the animal is generally required to return to the table on which it was just fed-a stay strategy. However, the three-table task can be arranged so that the correct response involves going to the unbaited table (i.e., a shift solution can be required). Under these conditions, the correct response on the test trial would be congruent with the predisposition to shift. Thus, two types of problems can be set up on the threetable task-the typical stay problem, in which the animal must return to the baited table, vs. a shift problem, in which the animal must go to the nonbaited table on the test trial.

The present experiment is concerned with whether the stay problem poses a more or less difficult requirement than the shift problem on the three-table task. The experiment simply compares the performance of subjects who are required to return to the place where they were fed (stay) with that of subjects required to go to the table that was not baited (shift) during the feeding phase. In addition, this experiment also considers the effect of allowing the animals to consume either all of the food available during the feeding phase or only a portion of the food. The latter condition is generally used when the three-table task is set up as a stay problem. However, in the radial-maze task, the animals generally consume all of the food in a given runway before leaving the runway for the next choice (Olton \& Samuelson, 1976).

\section{Method}

Subjects. Twenty-four Long-Evans strain male rats, obtained from the department breeding colony, were used in this experi- ment. The animals averaged 92 days of age upon receipt and weighed $389-452 \mathrm{~g}$. They were housed individually in a temperaturecontrolled colony room $\left(20^{\circ} \pm 2^{\circ} \mathrm{C}\right)$ under a 14-h (0700-2100) light cycle and a 10-h (2100-0700) dark cycle. Fourteen of the animals were used in Part A of the experiment, and 10 were used in Part B.

Apparatus. A version of the Maier three-table task was used that had three pathways (each $1.15 \mathrm{~m}$ long). These pathways formed equal angles from a center point. The tables at the end of each pathway differed in size, shape, and position in the room. Each table was faced with a wooden screen that hindered the observation of one table from another. Entrance to each table was provided by an $8 \times 8 \mathrm{~cm}$ hole cut in the wooden screen. The entire apparatus was painted a flat black and housed in a 3.4 $\times 3.4 \mathrm{~m}$ room. One side of the room consisted of a boarded-up window, while the other sides were lined with bookshelves, file cabinets, and a desk. However, no particular side of the room could be considered to be more salient than another. The apparatus and the room were diffusely illuminated by a $40-\mathrm{W}$ incandescent lamp located in one corner of the room.

Procedures. This experiment was conducted in two parts. In Part A, regardless of the problem confronting the animals (stay vs. shift), the animals were allowed to consume only a part of the food available during the feeding phase of the procedure. In Part B of the experiment, the animals were allowed to consume all of the $1 \mathrm{~g}$ of wet mash available during the feeding phase.

Prior to the initiation of testing in each part of the experiment, the animals were assigned randomly to one of two problem groups (stay or shift) and given $\mathbf{1 0} \mathrm{min}$ of handling for a 5 -day period. Following this 5 -day period, the animals were given a minimum of 5 days of training to run for food down a straight pathway similar in length and width to the pathways used on the three-table apparatus.

Following this pretraining period, the animals received one test session per day for 24 days. Each daily test session consisted of an initial 15-min exploratory period in which the animals were allowed to run freely over the entire unbaited apparatus. This phase was followed by a feeding period during which the interconnecting pathway system was disconnected from the tables and a dish of wet food was placed on one of the tables behind the wooden screen. The rats were placed on the table for a $1-$ min period and were allowed to eat either part of the food present or all of a 1-g ration placed in the feeding dish. In the latter instance, all but one animal consumed the $1 \mathrm{~g}$ of wet mash in the 1 -min feeding period. Three minutes after the completion of the feeding period, the test trial was given. During the period between feeding and the test trial, the animals were removed from the apparatus and placed in their holding cages, the runway system was replaced, and the apparatus was wiped down. The food dish was then placed either on the table where the rats had just been fed (stay) or on one of the remaining two tables (shift).

For the test trial, an individual animal was placed on one of the tables that was not baited during the feeding experience. This table served as the start table for that day. A correct response was scored when the animal ran directly from the start table to either the table that was baited during the feeding phase (stay problem) or to the other table, which was not baited during the feeding experience but which now served as the goal table for the shift-problem animals on the test trial. A different combination of start-goal tables was used each day in varying sequences over the four blocks of the six possible start-goal table combinations. If the animals did not run directly to their respective correct tables on the test trial, they were allowed to retrace their paths and make another choice. This second choice was generally correct.

\section{Results}

Figure 1 shows the performance of the animals in Part $A$ that were given a partial feeding during the feeding phase. In this and subsequent figures, each point represents the percentage correct on six trials averaged over animals. The figure shows that the 
animals tested on the stay problem (i.e., to return to the baited table of the feeding phase) performed from the beginning of testing almost at asymptote. In contrast, the animals given the shift problem required a number of days of testing before reaching the level of performance of the animals tested on the stay problem. A repeated measures analysis of variance, with type of problem as the between-groups factor and blocks of days as the repeated factor, revealed that, over all trials, stay-problem animals were more successful at solving their problem than shift animals were at their problem $[F(1,12)=14.4, p<$ $.003]$. A significant problem $\times$ blocks of trials interaction showed that the shift animals did make a gradual improvement in performance as testing continued $[F(3,36)=3.05, p<.05]$. By the final block of trials, there was no difference between the success rate of the shift-problem animals as compared with the stayproblem animals $[\mathrm{t}(12)=.74]$.

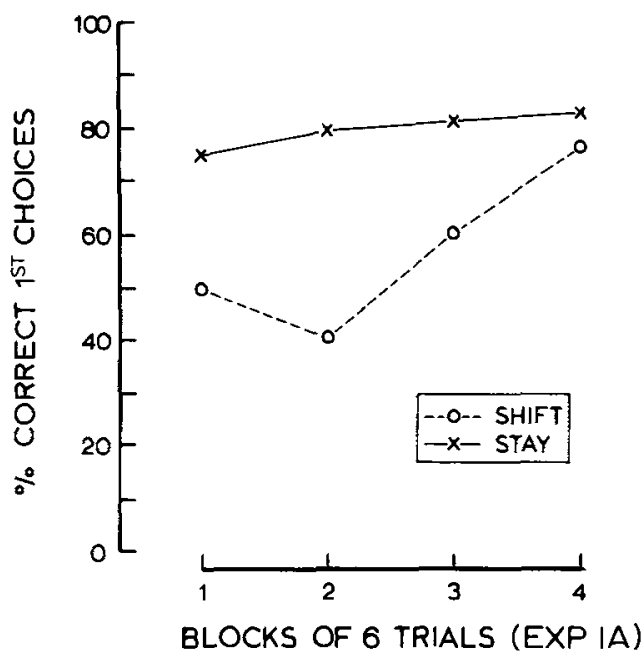

Figure 1. Performance of rats tested on either a stay or shift problem on the three-table task following partial feeding.

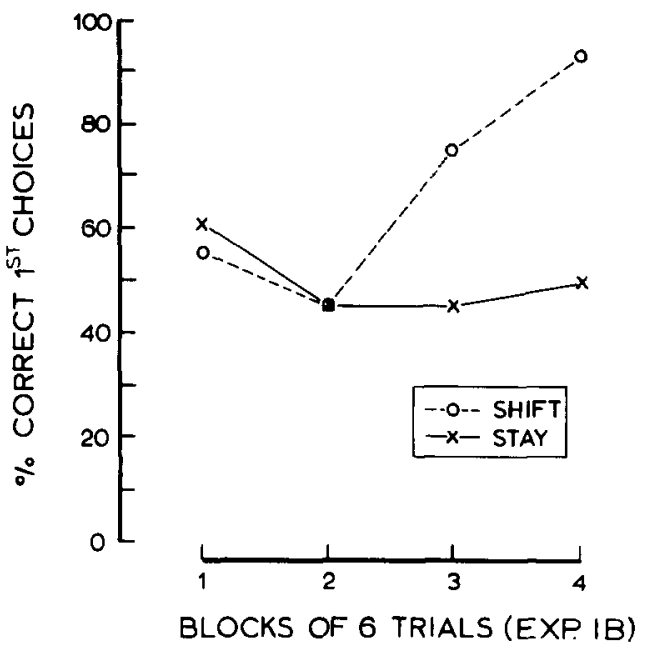

Figure 2. Performance on stay and shift problems following complete feeding during the feeding experience on the three-table task.
The results obtained in Part $B$, when the animals were allowed to consume all of the food placed on the baited table during the feeding phase of the procedure, are shown in Figure 2. In contrast with the data shown in Figure 1, it is apparent that the shift problem was solved, while the animals tested on the stay problem never performed above the chance level $(50 \%)$ despite repeated testing. A repeated measures ANOVA demonstrated a significant problem $x$ block of trials interaction $[F(3,24)=7.01, p<.005]$, which when further analyzed by a t test revealed a significant difference between the groups on the final block of trials $[t(8)=7.5, p<.01]$. There were no main effects of either problem or blocks of trials. Shift-problem animals were solving their problem at better than chance levels. The stay-problem animals, in contrast, showed no improvement in performance and tended to make the same directional turn at the choice point on better than $80 \%$ of their trials.

\section{Discussion}

A number of facts are apparent from this experiment. First, it is clear from Part $\mathbf{A}$ that the threetable task can be configured such that there are two distinct problems-a stay problem and a shift problem. Stay refers to the returning to the baited table on the test trial, while shift refers to not going to the table that was baited during the feeding phase. Second, it would appear that the shift problem is a more difficult problem for the animals, since, regardless of the kind of feeding (partial or complete), the shift solutions required a number of days of testing before they became dominant. In contrast, in the partial feeding condition (Part A), stay solutions appeared within the first block of test trials. The fact that shift solutions do not readily appear in this task, regardless of the kind of feeding, is contrary to what would be expected if rats have a predisposition not to return to a place where they just had found food. Thus, the appearance of shift solutions in the threetable task may reflect a type of psychological process different from a win-shift strategy on the radial arm maze. Finally, the fact that stay solutions appear only when the animals are given a partial feeding (Part A) indicates that the feeding experience that the animals are given prior to the test trial provides considerable information to the animals as to the availability of food. When the food is totally consumed during the feeding phase, stay solutions simply do not appear.

\section{EXPERIMENT 2}

Inasmuch as Experiment 1 indicated that shift solutions in the three-table task are relatively slow to come to expression, regardless of the nature of the feeding experience (partial or complete), it would indeed appear that performance on the three-table 
task is in fact not determined by the same type of process as is functional in the case of the radial arm maze, in which such solutions were readily acquired. However, there is a major difference between the testing procedures in the two tasks. It will be recalled that, in the radial-maze task, animals are not given a daily exploratory experience prior to being placed on the central platform from which the arms radiate. They are merely food-deprived and placed in the apparatus, free to do what they desire. In contrast, on the three-table task, the rats are allowed to explore the unbaited three tables and their interconnecting runways prior to the feeding phase each day. To what extent does the prior exploratory experience inhibit the appearance of the shift solution on this task? The second experiment was concerned with determining whether omission of the exploratory experience would facilitate performance on the shift problem on the three-table task. As with the first experiment, Experiment 2 was also carried out in two parts. In Part A, the animals received a partial feeding experience and, in Part B, a complete feeding.

\section{Method}

Subjects. Twenty Long-Evans male rats of the same ages as those in Experiment 1 served as subjects. Ten animals were used in Part A and 10 in Part B. As in Experiment 1, the animals used in each part of this experiment were also assigned randomly to each of the two types of problems.

Apparatus. The same apparatus used in Experiment 1 was used in this experiment.

Procedure. All preliminary training was similar to that carried out in Experiment 1. Following the completion of the preliminary training, the test sessions began. The test session consisted of only two phases. First, there was the feeding phase on one of the tables, and then there was the test trial that was carried out as described in Experiment 1. In neither Part A nor Part B were the animals allowed to explore the apparatus prior to the feeding. Thus, the test session consisted of a 1 -min feeding on one of the tables and the test trial. Here, too, as in Experiment 1, the animals in Part A received a partial feeding in that they did not consume all of the food available, while the animals in Part B were given $1 \mathrm{~g}$ of wet mash that could be totally consumed during the 1-min feeding period. In this experiment, the animals were given one test session per day for 42 days.

\section{Results}

As can be seen from Figure 3 in Part A, none of the animals in either group were successful on their respective problems. Both stay and shift animals performed at the $50 \%$ level. Analysis of variance revealed no significant difference between the two groups $[F(1,8)=1.98]$ and no systematic change over blocks of test trials $(F<1)$. However, the animals in both groups were not simply choosing a table at random on the test trial. Their pattern of behavior indicated that they were using what we have previously identified as a turn strategy (Herrmann, Black, Doherty, \& Ellen, 1980). Shift-problem animals, on $77.6 \%$ of their tests, and stay-problem animals, on $77.2 \%$ of their tests, made the same directional turn at the center point of the $Y$. This type of consistent

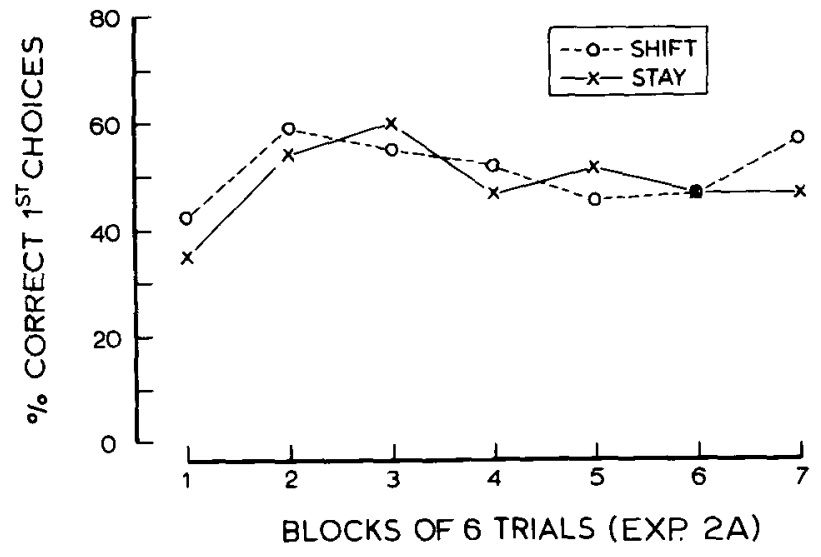

Figure 3. Performance following partial feeding on stay and shift problems in the absence of a prior exploratory experience.

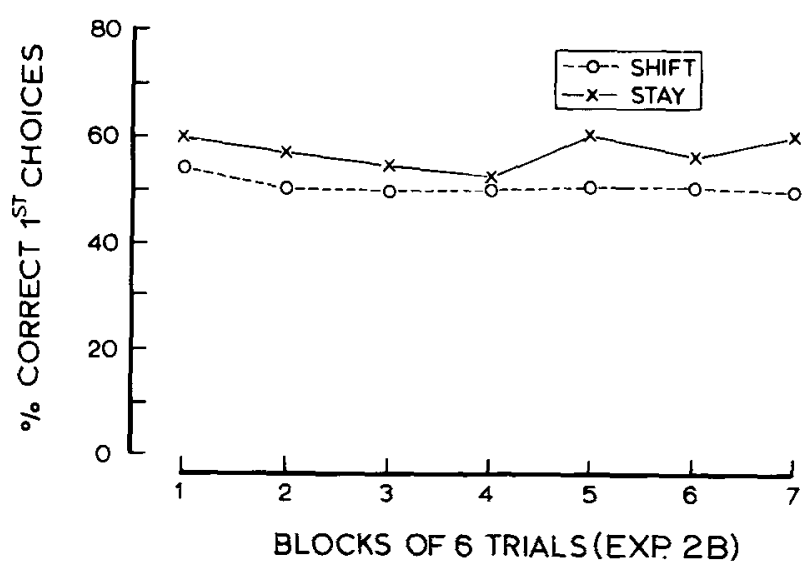

Figure 4. Performance on stay and shift problems following complete feeding in the absence of exploration.

turn strategy results in successful food acquisition within two choices on each trial, regardless of the kind of problem (i.e., stay vs. shift).

Figure 4 shows the results obtained in Part B when the animals on both problems were allowed to completely consume the food during the feeding period. Here, too, it can be seen that neither the stay- nor the shift-problem animals were able to solve their respective problems and that neither group was superior to the other $[F(1,8)=2.51]$. The animals in both groups displayed turn strategies on more than $70 \%$ of their test trials. Thus, these data are in no way different from those of Part A, in which the animals were allowed to consume only a portion of their food during the feeding period.

\section{Discussion}

The results of Experiment 2 are quite clear in showing that, regardless of the nature of the feeding experience (partial vs. complete), animals not allowed to explore the apparatus prior to the feeding experience simply do not solve either the stay or the 
shift problem. Thus, omitting the exploratory phase from the procedure does not allow shift solutions to come to expression. The appearance of consistent turn strategies in both parts of this experiment suggests that when animals are placed in an unexplored and hence unfamiliar environment, they tend to rely on body turns to bring them to food. Thus, these findings contrast sharply with those seen with the radial arm maze. On that task, despite the lack of a daily exploratory period, animals readily shift their choices from one alley to another as they find food in an alley.

\section{GENERAL DISCUSSION}

The results of these experiments clearly indicate that, while a spatial memory containing the representations of the spatial relations existing among the tables is necessary for performance on the three-table task, it is not sufficient. The type of feeding experience determines whether a stay problem will be solved. If the animals receive a partial feeding experience, they can solve the stay problem. In contrast, the shift problem can be solved regardless of the kind of feeding; however, the shift-problem solution will be delayed relative to the appearance of the stayproblem solution. That is, 18-24 trials are required before the shift problem can be solved, while the stay problem is solved within the first block of six trials. This latter fact would suggest that the shift problem on the three-table task is more difficult than the stay problem and that the shift solution is not simply the reflection of a predisposition not to return to a place where food had just been found. In this sense, the shift solution on the three-table task would reflect a process different from that operating in the radial arm maze (Olton \& Samuelson, 1976).

It should be noted that stay solutions did not appear when there was a total consumption of the food during the feeding phase. It is evident from this result that the feeding experience provides information to the animal not only as to the location of food, but also as to its availability. However, it would also seem that neither type of solution (stay or shift) appears unless there has been a prior exploratory period. This latter finding has been reported earlier (Stahl \& Ellen, 1974); it suggests that before an animal can learn from the feeding experience that a given table has food on it, the animal must first learn where that table is in relation to the other tables. It would appear that the animals do not learn the spatial relations existing between the tables when they are running from the start table to one of the goal tables on the trial, as occurred in Experiment 2. Maier (1932b) has shown that an animal does not learn that $A$ is spatially related to $B$ simply by running from $A$ to $B$. The animal must also run from B to $A$ soon thereafter. In Experiment 2, although the animals ran from $A$ to $B$, for example, on the test trial of a given day, they may not have run from $B$ to $A$ until several days later on another test trial. Thus, the possibility exists that the opportunity to learn that $\mathbf{A}$ is related to $B$ was simply not available to the animals in Experiment 2, in which no prior exploratory period had been given. However, once the animals have learned the spatial relations existing among the tables, and once the memorial representations of these relations have been reactivated by the exploratory experience, then the feeding phase can function to inform the animal as to the locus and availability of food. Of interest in this condition are our observations that when the feeding experience is given prior to the exploratory experience, animals cannot perform successfully on a stay problem on the threetable task (Herrmann, Doherty, \& Ellen, Note 1). Thus, it would appear that the exploratory phase allows the animals to learn the spatial relationships among the tables and that the daily use of exploration allows the memory of these relations to be reactivated.

From this perspective, it would appear that successful performance of either the stay or shift problem on the three-table task involves the integration of two elements of information. The first has to do with the spatial relationships of the tables, while the second involves the daily locus and availability of food. If we assume that information about the spatial relations that exist among the tables is stored in reference memory (Honig, 1978) and reactivated by the exploratory phase, and the information as to the daily locus and availability of food is temporarily stored in working memory (Olton, Becker, \& Handelmann, 1979), then it would appear that the successful performance on either problem on the three-table task reflects the integration of information from both memory stores. Moreover, since the information in working memory is changed on a daily basis, the appearance of a solution on any given day reflects the reorganization of these memorial representations.

There is another, related, issue that needs to be addressed. The findings of Experiment 2 are at variance with those of Olton et al. (1980) demonstrating that rats tested on a version of the three-table task could solve both stay and shift problems despite the fact that there was no daily exploratory period. They reported that the stay solution was a more difficult one for the animals to acquire. Unfortunately, information is not available from their report on the availability and salience of extramaze cues in the testing environment. The possibility exists that extramaze stimuli, if they have been consistently associated with feeding, can allow animals to solve stay problems on the three-table task in the absence of a prior exploratory phase. Herrmann et al. (1980) demonstrated that even though rats did not receive a prior daily exploratory experience, they could return 
to the baited table on the test trial, provided a light source was present during the feeding phase and the test trial. Thus, the success of Olton et al. (1980) in obtaining stay or shift solutions when testing the animals on the three-table task without exploration may have stemmed from the fact that there were sufficient extramaze cues in the room that could have functioned to identify the baited table each day. However, it would follow that such performance does not reflect integrative solutions of the type characteristically found on the three-table task. Rather, the performance reported by Olton et al. was simply reflecting some sort of S-R strategy or "taxon" (O'Keefe \& Nadel, 1978) hypothesis, such as to approach or avoid a particular cue associated with food. In this connection, Hermann et al. (1980) showed that if rats received a prior exploratory experience in addition to being fed and tested on a table that was marked by a light cue, then the stay solutions did not come to expression. In this instance, the two types of learning apparently could not be integrated to allow for the appearance of stay solutions. At any event, the differences obtained by Olton et al. (1980) and the present results cannot be unequivocally resolved at this time and remain an issue for further investigation.

In contrast with the three-table task, performance on the radial arm maze does not seem to reflect the integration of information stored in reference and working memory. Although there is a reference memory component present in the radial-arm-maze task (i.e., food is at the end of each arm, etc.), the critical factor determining performance on this task would seem to be the working memory factor. The rats must identify and remember for a few minutes which arms of the maze have been entered and which have not. Apparently, extramaze stimuli suffice for defining an arm's location in the testing environment (Olton, Collison, \& Werz, 1977; Olton \& Samuelson, 1976; Suzuki, Augerinos, \& Black, 1980). While there are a number of strategies that can be stored in reference memory and that could function to control performance (i.e., choose adjacent arms, chain responses, follow odor trails or other intramaze cues), such reference memory material does not seem to underlie the rapidity with which the radial maze task is mastered (Olton \& Samuelson, 1976). Rather, the accurate memory for places where food has been found, together with a predisposition to shift following the finding of food, are all that are necessary to account for the rapidity of acquiring the winshift strategy on the radial arm maze. To this extent, it would appear that, although spatial memory is required on both the radial arm maze and the threetable task, in the radial arm maze the rapid learning of the shift strategy comes about because the optimal strategy is congruent with the animal's predisposition to shift. Thus, the win-shift strategy that is learned on the radial arm maze would not reflect the same kind of process as a win-shift solution on the threetable task, in which elements of information in working memory must be integrated with information in reference memory.

Throughout this paper, reference has been made to the rat's predisposition to shift its choice once it has found food in one location. Olton et al. (1980) and Olton and Samuelson (1976) imply that this predisposition to shift may be a foraging strategy that has become a species characteristic of the rat. Our results indicate that shift solutions are favored relative to stay solutions only when animals completely consume all of the food present in a given location. Under conditions of partial feeding, stay solutions are favored. This finding would suggest that it is the availability of food in a given location, rather than some species characteristic, that predisposes the animal to look toward another location following feeding. In the radial arm maze, the animals completely consume all of the food in a given arm, leaving none remaining in that arm. Thus, the rapidity with which the win-shift strategy is adopted in the radial arm maze may be not so much the result of a species-characteristic foraging behavior that happens to be compatible with the optimal strategy as a simple reflection of the fact that animals do not return to places where food is no longer available. Thus, resorting to an ethological explanation to account for the rapid acquisition of the win-shift strategy in the radial arm maze is unnecessary since there is nothing within that task that cannot be accounted for in terms of the procedures employed.

\section{REFERENCE NOTE}

1. Herrmann, T., Doherty, D., \& Ellen, P. Places vs, routes in spatial problem-solving. Unpublished manuscript, 1978.

\section{REFERENCES}

Herrmann, T., Black, A. H., Doherty, D., \& Ellen, P. Visual cues fail to attenuate deficits on a spatial-integration task following septal or fornical damage. Physiological Psychology, 1980, 8, 29-32.

Honig, W. K. Studies of working memory in the pigeon. In S. H. Hulse, H. F. Fowler, \& W. K. Honig (Eds.), Cognitive aspects of animal behavior. Hillsdale, N.J: Erlbaum, 1978.

Maler, N. R. F. The effects of cerebral destruction on reasoning and learning in rats. Journal of Comparative Neurology, 1932, 54, 47-55. (a)

MAIER, N. R. F. A study of orientation in the rat. Journal of Comparative Psychology, 1932, 14, 387-399. (b)

O'KeEFe, J., \& NadeL, L. The hippocampus as a cognitive map. Oxford: Clarendon Press, 1978.

Olton, D. S. Characteristics of spatial memory. In S. H. Hulse, H. F. Fowler, \& W. K. Honig (Eds.), Cognitive aspects of animal behavior. Hillsdale, N.J: Erlbaum, 1978.

Olton, D. S. Mazes, maps and memory. American Psychologist, $1979,34,583-596$.

Olton, D. S., Becken, J. T., \& Handelmann, G. E. Hippocampus, space, and memory. The Behavioral and Brain Sciences, $1979,2,313-365$. 
Olton, D. S., Collison, C., \& Werz, M. A. Spatial memory and radial arm maze performance of rats. Learning and Motivation, 1977, 8, 289-314.

Olton, D. S., Handelmann, G. E., \& Walker, J. A. Characteristics of spatial memory and optimal foraging in rodents. In A. Kamil \& T. Sargent (Eds.), Optimal foraging. New York: Garland/STPM, 1980.

Olton, D. S., \& Samuelson, R. J. Remembrance of places passed: Spatial memory in rats. Journal of Experimental Psychology: Animal Behavior Processes, 1976, 2, 97-116.

Olton, D. S., \& Schlosberg, P. Food searching strategies in young rats: Win-shift predominates over win-stay. Journal of
Comparative and Physiological Psychology, 1978, 92, 609-618. STAHL, J. M., \& ElLEN, P. Factors in the reasoning performance of the rat. Journal of Comparative and Physiological Psychology, 1974, 87, 598-604.

Suzuki, S., Augerinos, G., \& Black, A. H. Stimulus control of spatial behavior on the eight-arm maze in rats. Learning and Motivation, 1980, 11, 1-18.

Winocur, G. The hippocampus and cue utilization. Physiological Psychology, 1980, 2, 280-288.

(Manuscript received March 27, 1981; revision accepted for publication August 30, 1981.) 